\title{
Project Title: Enhanced Power Stability for Proton Conducting Solid Oxides Fuel Cells
}

Report Title: First principles modeling of yttrium-doped $\mathrm{BaZrO}_{3}$ solid electrolyte

Type of Report: Semi-Annual Technical Progress Report

Reporting Period Start Date: September 30, 2002

Reporting Period End Date: March 30, 2003

Principle Authors: Claudio O. Dorso, Boris V. Merinov, and William A. Goddard III (PI)

Date Report was Issued: April 30, 2003

DOE Award Number: DE-FC26-02NT41631

Name and Address of Submitting Organization: Materials and Process Simulation Center, California Institute of Technology, mail code 139-74, 1200 E. California Blvd., Pasadena, CA 91125 


\section{DISCLAIMER}

This report was prepared as an account of work sponsored by an agency of the United States Government. Neither the United States Government nor any agency thereof, nor any of their employees, makes any warranty, express or implied, or assumes any legal liability or responsibility for the accuracy, completeness, or usefulness of any information, apparatus, product, or process disclosed, or represents that its use would not infringe privately owned rights. Reference herein to any specific commercial product, process, or service by trade name, trademark, manufacturer, or otherwise does not necessarily constitute or imply its endorsement, recommendation, or favoring by the United States Government or any agency thereof. The views and opinions of authors expressed herein do not necessarily state or reflect those of the Unites States Government or agency thereof. 


\begin{abstract}
$\mathrm{Ab}$ initio Quantum mechanics calculations of the equation of states for $\mathrm{BaZrO}_{3}$ have been performed and the bulk modulus has been obtained. The value of the modulus is in good agreement with reported experimental values. Equilibrium proton positions in $\mathrm{Y}$-doped $\mathrm{BaZrO}_{3}$ with dopant concentrations from 12.5 to $50 \%$ were investigated. Initial rough estimates of the transition barriers have been made. Our results suggest that the proton migration pathway may involve secondary minima with two maxima (symmetric with respect to the center of the path).

In the next phase of this project the results of our quantum mechanical calculations will be used to develop a new Reactive Force Field (ReaxFF) based on first principles. This Reactive Force Field will be used for much molecular dynamics simulations or much larger systems to investigate proton migration in bulk and surface regions of fuel cells.
\end{abstract}




\section{TABLE OF CONTENTS}

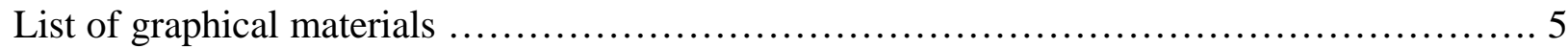

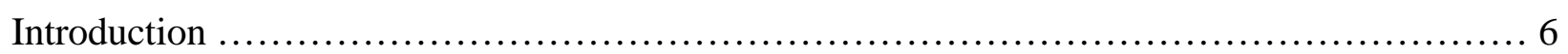

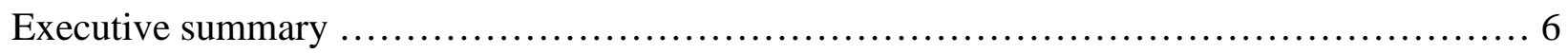

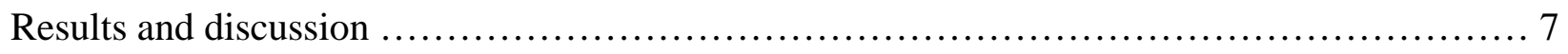

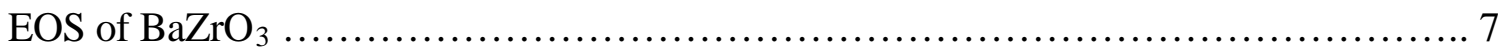

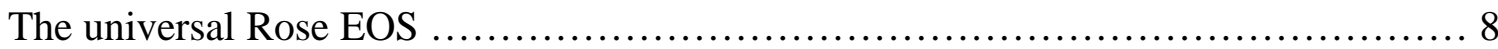

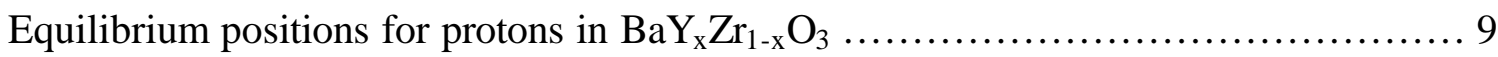

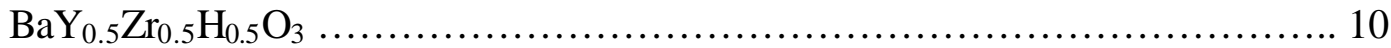

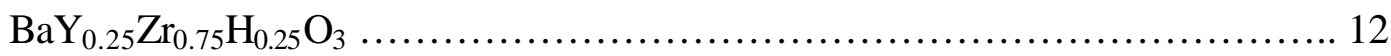

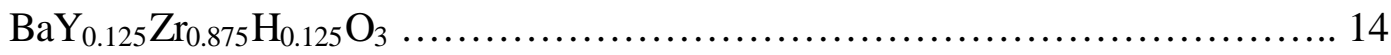

First estimation of transition barriers ........................................... 15

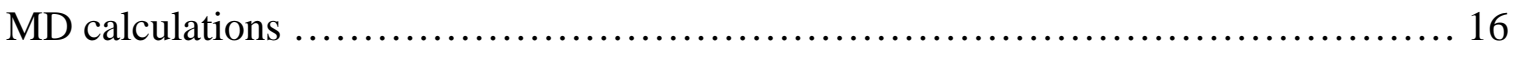

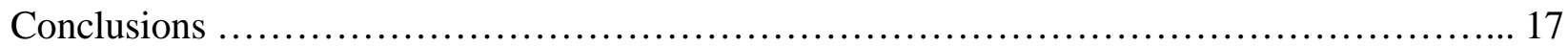

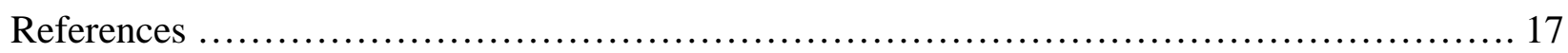

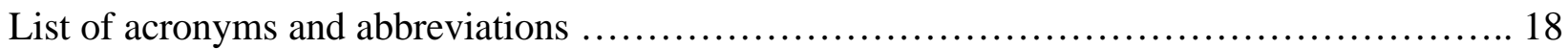




\section{LIST OF GRAPHICAL MATERIALS}

Fig.1. EOS for cubic $\mathrm{BaZrO}_{3}$.

Fig.2. Experimental and calculated bulk modulus for $\mathrm{BaZrO}_{3}$.

Fig.3. Schematic representation of the equilibrium positions investigated in this work.

Fig.4. The asymptotic configuration after QM relaxation (configuration 1).

Fig.5. Values of the relative energies of the different relaxed configurations obtained.

Fig.6. Schematic representation of the relaxed states found in this calculation.

Fig.7. Energy differences for the $2 \times 2 \times 1$ case.

Fig.8. The lowest energy configuration for $\mathrm{BaY}_{0.25} \mathrm{Zr}_{0.75} \mathrm{H}_{0.25} \mathrm{O}_{3}$.

Fig.9. Schematic representation of the relaxed states considered for $\mathrm{BaY}_{0.125} \mathrm{Zr}_{0.875} \mathrm{H}_{0.125} \mathrm{O}_{3}$.

Fig.10. Energy differences for $\mathrm{BaY}_{0.125} \mathrm{Zr}_{0.875} \mathrm{H}_{0.125} \mathrm{O}_{3}$.

Fig.11. Relaxed configurations for $\mathrm{BaY}_{0.125} \mathrm{Zr}_{0.875} \mathrm{H}_{0.125} \mathrm{O}_{3}$.

Fig.12. Relative energies of the relevant states along the path on the edge of the oxygen octahedron parallel to the Y plane.

Fig.13. The main characteristics of the states. 


\section{INTRODUCTION}

Y-doped $\mathrm{BaZrO}_{3}$ is one of the most promising proton conducting materials for solid oxide fuel cell (SOFC) applications. It has desirable properties such as high protonic conductivity and excellent chemical and mechanical stability. The current limitation for application of Y-doped $\mathrm{BaZrO}_{3}$ in SOFC is the extremely high grain boundary resistance, which leads to the relatively poor total conductivity.

In order to understand the conductivity (both bulk and grain boundary) as well as the role of defects and dopants in transport at the molecular level, we have designed the following first principles based multi-scale strategy:

1. Accurate first principles Quantum Mechanical (QM) calculations of the materials under analysis: equations of state (EOS), energetics of various configurations of dopant-protons, migration barriers, etc.

2. Derive a First Principles-based Reactive Force Field (ReaxFF). ReaxFF uses the QM data described in 1. It allows large-scale Molecular Dynamics (MD) simulations that enable the study of proton transport under realistic conditions (temperature, structure, etc.) as well as the role of grain boundaries, defects, etc.

3. Mesoscale Kinetic Monte Carlo (KMC) simulations based purely on the results of 1 and 2 .

According to the project schedule QM calculations on model structures have to be performed during the first year. In this semi-annual technical progress report we present ab initio results (DFTGGA) that allows us to develop relationships between structures and energetics. Such calculations represent the fundamental data on which ReaxFF and KMC will be based.

\section{EXECUTIVE SUMMARY}

We have performed ab initio calculations of the EOS of $\mathrm{BaZrO}_{3}$ from which a bulk modulus has been obtained. This bulk modulus is in very good agreement with the reported experimental value. We have investigated the equilibrium positions of protons in Y-doped $\mathrm{BaZrO}_{3}$ for three concentrations of the dopant. Characteristic features of the equilibrium positions agree with those obtained from previous calculations [1]. A general trend for the maximum energy differences could be described as decreasing as the concentration decreases in the range considered. These results seem to confirm the assumption that the high concentration of the dopant might lead to a trapping of the protons [2].

Finally, we have made our first estimation of transition barriers. It is interesting to note that our initial results based on ab initio calculations we find that the overall shape of the energy difference landscape has two maxima (symmetric with respect to the center of the path). This is in contrast with recent calculations [2] in which the oxygen configuration was assumed to be frozen and the corresponding transition barrier displayed just one maximum at the center.

\section{RESULTS AND DISCUSSION}


Our QM calculations of $\mathrm{BaZrO}_{3}$ and $\mathrm{BaY}_{\mathrm{x}} \mathrm{Zr}_{1-\mathrm{x}} \mathrm{O}_{3}$ are based on Density Functional Theory (DFT) [3,4] using Generalized Gradient Approximation (GGA) [5] to treat the exchange-correlation energy functional. In these calculations pseudo-potentials are used to replace the core electrons. All calculations have been performed at $\mathrm{T}=0 \mathrm{~K}$. We used the pseudo-potential local basis set code SeqQuest.

For $\mathrm{BaZrO}_{3}$ we studied EOS (i.e. the functional relationship between the energy and volume) and calculated the bulk modulus, which we compared with available experimental data.

For the doped material, $\mathrm{BaY}_{\mathrm{x}} \mathrm{Zr}_{1-\mathrm{x}} \mathrm{O}_{3}$, we analyzed the proton equilibrium positions for various values of the dopant concentration $\mathrm{x}$.

\section{$\mathrm{EOS}_{\text {of }} \mathrm{BaZrO}_{3}$}

We have studied the behavior of the energy (and as a consequence the behavior of the pressure $\mathrm{P}=-$ $\mathrm{dE} / \mathrm{dV}$ ) as a function of the volume for cubic $\mathrm{BaZrO}_{3}$, We have also explored the stability of this state against deformations at constant volume (tetragonal and rombohedral). Unlike other perovskites we confirm that the cubic geometry is the most stable as suggested before from the experimental data and theoretical calculations.

Fig.1 shows the EOS, $[\mathrm{E}(\mathrm{V}), \mathrm{T}=0 \mathrm{~K})]$ for cubic $\mathrm{BaZrO}_{3}$. The results of the $\mathrm{QM}$ calculations are denoted by full circles while the curve is the corresponding fit according to the Rose EOS [2].

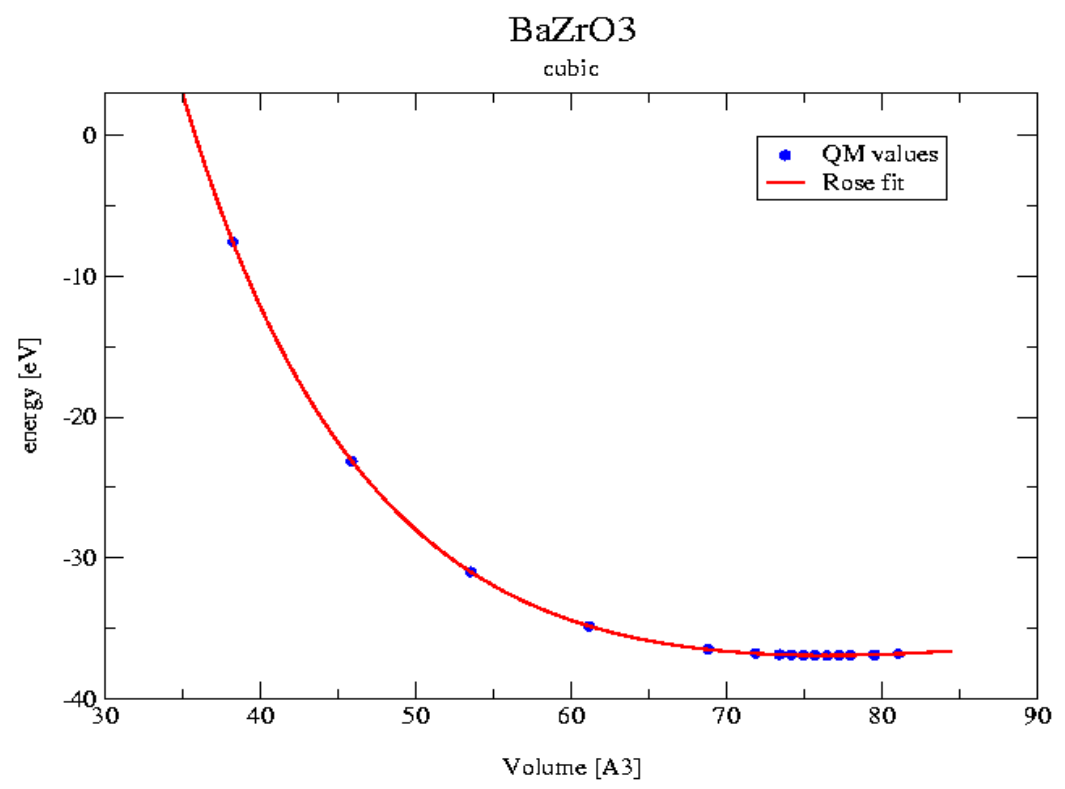

Fig.1. EOS for cubic $\mathrm{BaZrO}_{3}$. 


\section{The Universal Rose EOS}

The universal EOS of Rose is given by [2]

$$
E\left(r_{w s}\right)=\Delta E E^{*}\left(a^{*}\right)
$$

is the binding energy per atom and $r_{w s}$ is the radius of the Wigner Seitz sphere containing on the average one atom volume.

$$
a^{*}=\left(r_{w s}-r_{w s e}\right) /\left(r_{w s} \lambda\right)
$$

With $r_{w s}$ the equilibrium radius and 1 is a length scale.

$$
E^{*}\left(a^{*}\right) \approx f^{*}\left(a^{*}\right) e^{\left(-a^{*}\right)}
$$

With

$$
f^{*}\left(a^{*}\right)=f_{0}^{*}-f_{1}^{*} a^{*}+f_{2}^{*} a^{* 2}+f_{3}^{*} a^{* 3}
$$

Subject to the conditions that $E^{*}(0)=1 \cdot E^{*^{*}}(0)=0, E^{*^{\prime \prime}}(0)=1$, which then imply that $f_{0}^{*}=-1, f_{1}^{*}=-1, f_{2}^{*}=0$. From the least square fit of the ab initio data the following values for the free parameters are obtained:

$$
\begin{gathered}
\Delta \mathrm{E}=36.9076+/-0.0003 \\
\mathrm{r}_{\mathrm{wse}}=4.2441+/-0.0002 \\
\lambda=0.2438+/-0.0002 \\
\mathrm{f}_{3}{ }^{*}=0.1093+/-0.0013
\end{gathered}
$$

From this the bulk modulus can be calculated

$$
B=-V \frac{d P}{d V}=V\left(\frac{d^{2} E}{d V^{2}}\right)
$$

We obtain $B=144.597$ GPA

Fig. 2 shows this result (blue circle) together with the experimental values (red circles) as extracted from the NIST data base (red circles). As it can be seen from this figure, the agreement between the experiment and theory is quite satisfactory. 


\section{Bulk Modulus BaZrO3}

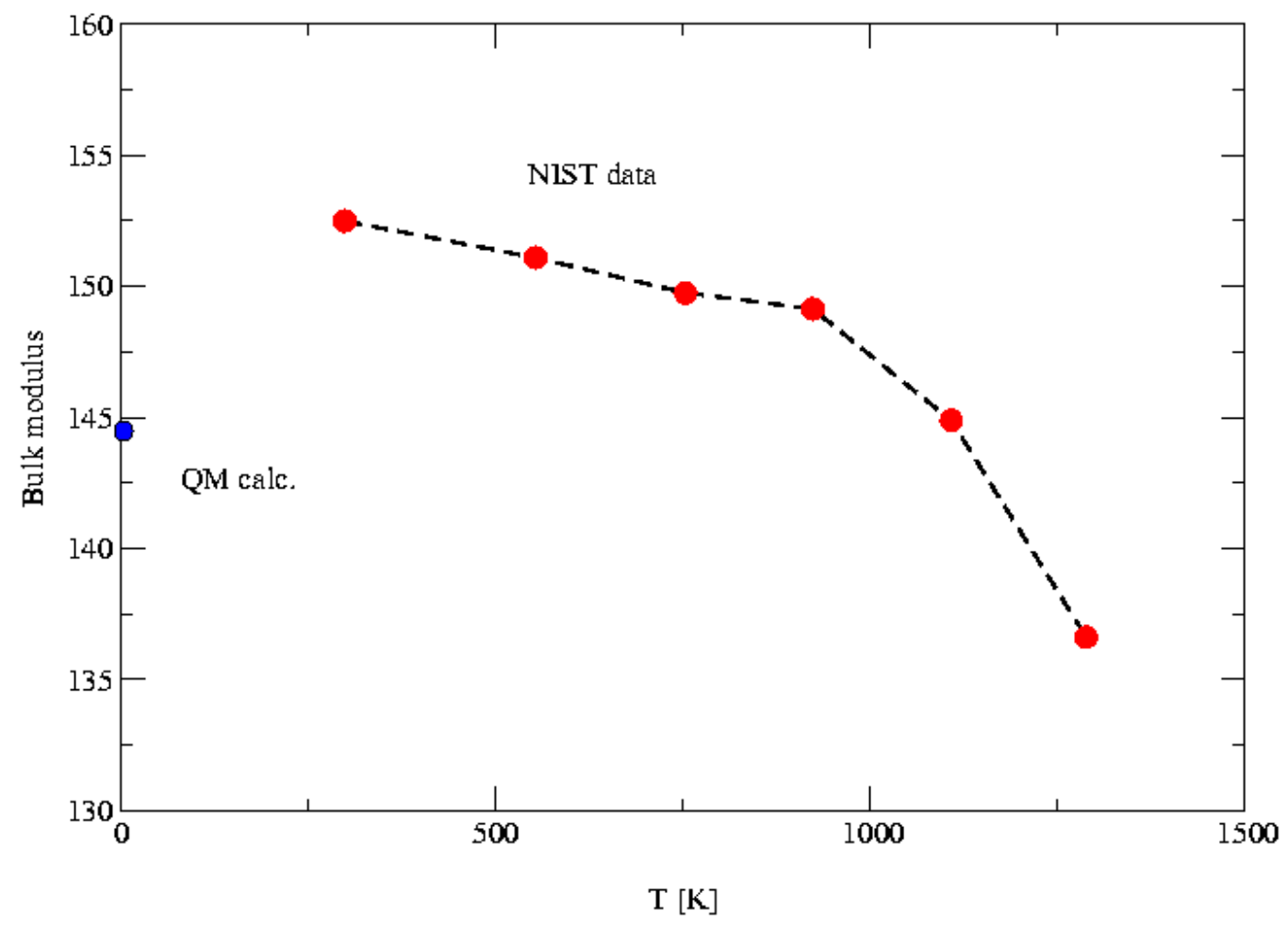

Fig.2. Experimental and calculated bulk modulus for $\mathrm{BaZrO}_{3}$.

\section{Equilibrium Position for Protons in $\mathrm{BaY}_{x} \mathrm{Zr}_{1-x} \mathrm{O}_{3}$}

Once the fundamental interactions of the base material were calculated and the accuracy of the method was established, we could focus on the energetics of $\mathrm{BaY}_{\mathrm{x}} \mathrm{Zr}_{1-\mathrm{x}} \mathrm{O}_{3}$. These calculations are very expensive, therefore, we limited the values of $\mathrm{x}$ to $0.5,0.25$ and 0.125 . The lowest concentration of Y corresponds to 8 unit cells with a total number of atoms equal to 41 .

These calculations pursue a double object. On the one hand, we need to generate accurate determination of the energies, positions and electronic populations (Mulliken analysis) to build an appropriate training set for determination of parameters for the ReaxFF. Once ReaxFF is developed, we will be able to perform large scale MD simulations. On the other hand, this information provides valuable insight into the possible relevant states for the protonic conduction process. Moreover, these data are relevant (together with the MD analysis) for determination of transition probabilities for the implementation of dynamical codes for KMC calculations, which will be used for the analysis of the system at mesoscopic scales.

$\mathrm{BaY}_{0.5} \mathrm{Zr}_{0.5} \mathrm{H}_{0.5} \mathrm{O}_{3}$

$\mathrm{BaY}_{0.5} \mathrm{Zr}_{0.5} \mathrm{H}_{0.5} \mathrm{O}_{3}$ has the highest dopant concentration analyzed in this work. This concentration $(\mathrm{x}=0.5)$ is much higher than the experimentally obtained optimal concentration $(\mathrm{x} \sim 0.15)$, which 
provides the highest conductivity. Such a small value of the concentration would preclude a simple picture of the conduction, i.e. percolation of Y sites. Such a percolation has a threshold of 0.31, i.e. by a factor 2 larger than the optimal concentration, so the mechanism of proton conduction is much more subtle.

Our model consists of parallel pure $\mathrm{Y}$ and pure $\mathrm{Zr}$ layers. Fig. 3 shows a schematic representation of the equilibrium positions obtained in our calculations (see the figure caption for details).

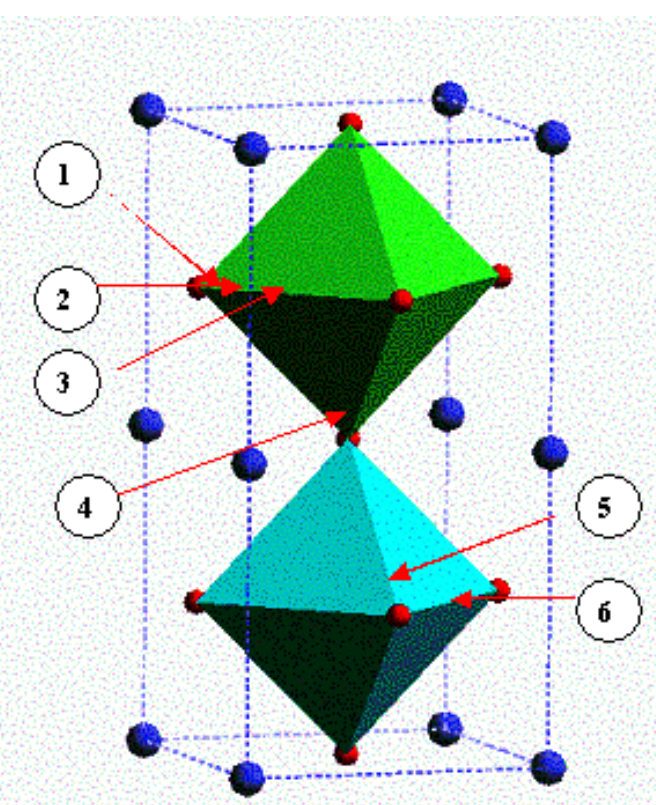

Fig.3. Schematic representation of the equilibrium positions investigated in this work.

Corresponding values of the energy are shown in Fig.5. All final configurations were obtained by allowing the system to fully relax, except for configuration 2 in which the distance between the proton and the nearest $\mathrm{O}$ was fixed to $1.07 \mathrm{~A}$. The $\mathrm{YO}_{6}$-octahedron is green and the $\mathrm{ZrO}_{6}$ octahedron is light blue.

An example of the actual final configurations is shown in Fig.4. One can see that the equilibrated proton is not aligned directly along the $\mathrm{O}-\mathrm{O}$ connection line of the corresponding $\mathrm{YO}_{6}$-octahedron but rather turned to the outside. This is a common feature of all our results. 


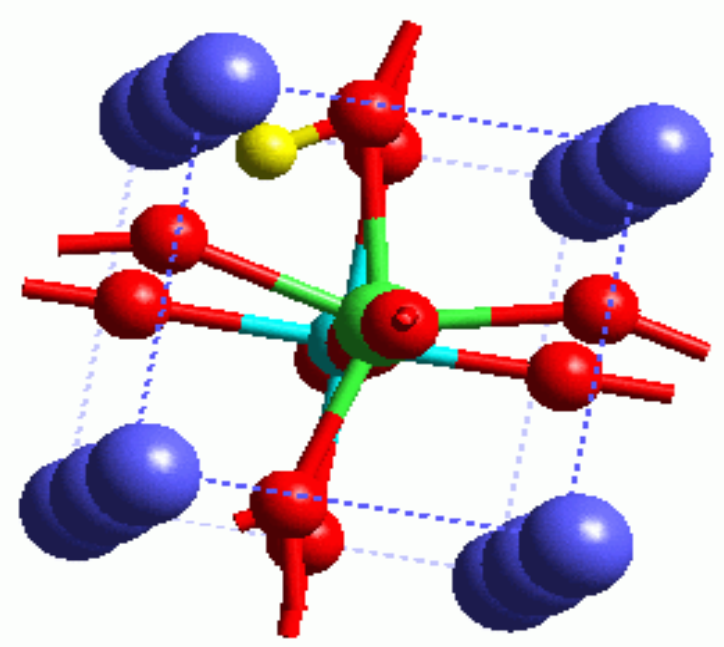

Fig.4. The asymptotic configuration after QM relaxation (configuration 1).

The $\mathrm{Y}$ atom is green, $\mathrm{O}$ - red, $\mathrm{Zr}$ - light blue, $\mathrm{Ba}$ - dark blue, the proton is yellow.

Finally, Fig.5 shows the values of the system energy referred to the minimal one (this scheme is used for all concentrations and the minimum-energy value is always referred to 1 in the schematic representations). As it can be seen, the difference between the minimum and the maximum is of order of $0.8 \mathrm{eV}$, which means that the height of the energy of saddle point of the transition state (assuming a direct path linking this two states) is larger than this value.

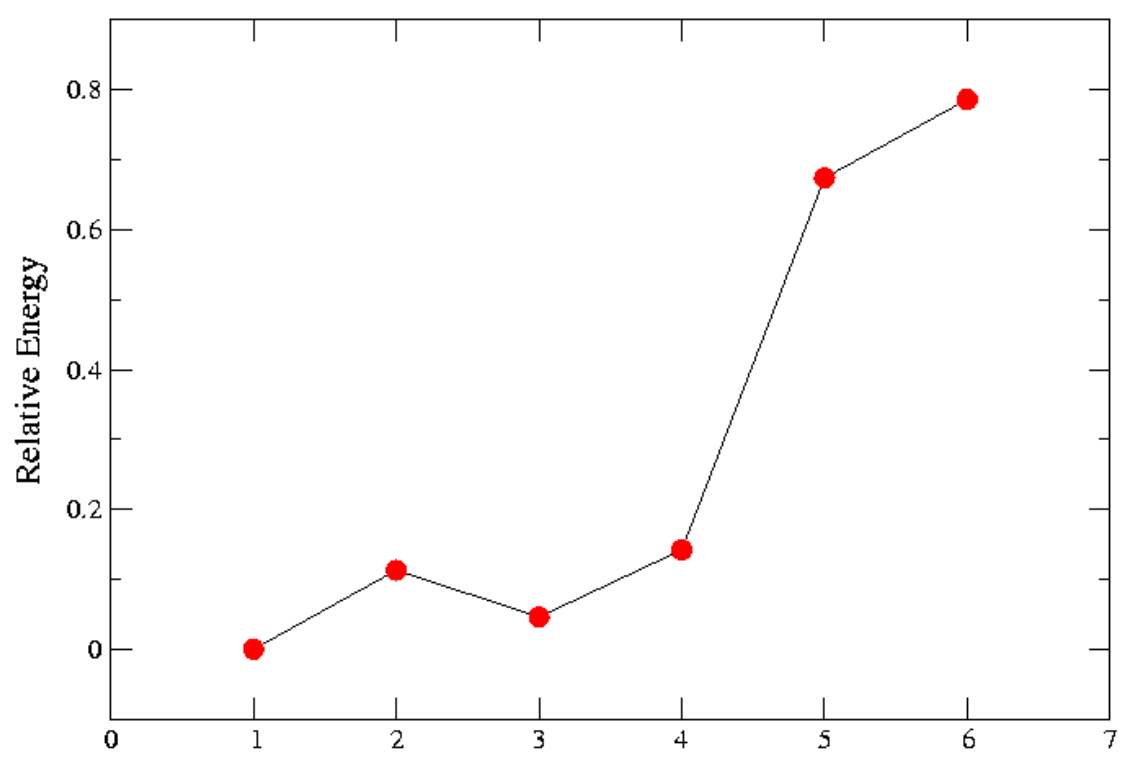


Fig.5. Values of the relative energies of the different relaxed configurations obtained. The first three values correspond to the proton being located between the neighboring $\mathrm{O}$ anions along the edge of the $\mathrm{YO}_{6}$ octahedron parallel to the plane containing the $\mathrm{Y}$ atoms.

\section{$\mathrm{BaY}_{0.25} \mathrm{Zr}_{0.75} \mathrm{H}_{0.25} \mathrm{O}_{3}$}

In the investigated geometries of $\mathrm{BaY}_{0.25} \mathrm{Zr}_{0.75} \mathrm{H}_{0.25} \mathrm{O}_{3}$ yttrium is ordered along the $\mathbf{y}$ direction in one dimensional strings so that each $\mathrm{Y}$ is surrounded by two aligned $\mathrm{Zr}$ along the $\mathbf{y}$ direction and four $\mathrm{Zr}$ along the $\mathbf{x}$ and $\mathbf{z}$ directions (see Fig.6).

Once again, we first present the schematic description of the positions of the relaxed protons (Fig.6), then a characteristic relaxed state (Fig.7) and finally the relative energies (Fig.8). It should be noted that in this case the difference in energy between the minimum and the maximum is of order of $0.4 \mathrm{eV}$, by a factor 2 lower than in the previous case.

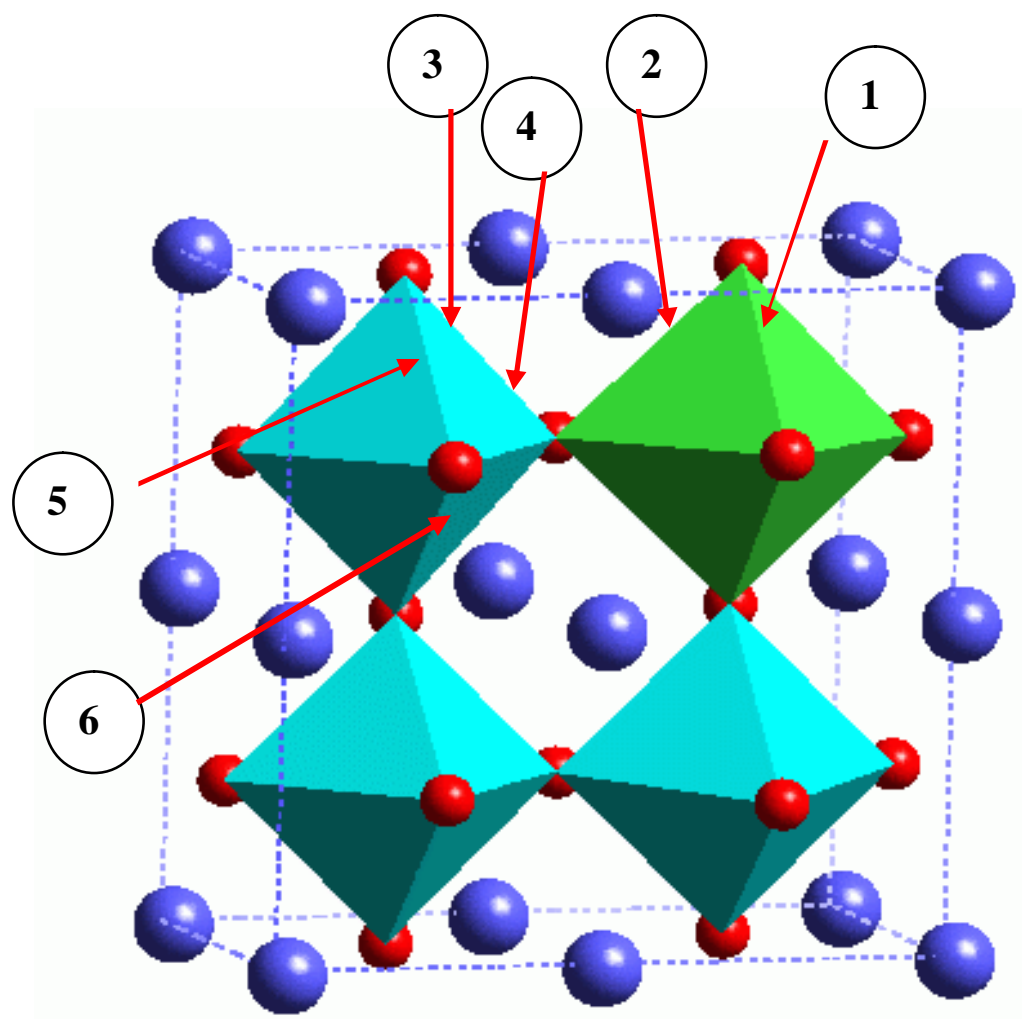

Fig.6. Schematic representation of the relaxed states found in this calculation. 


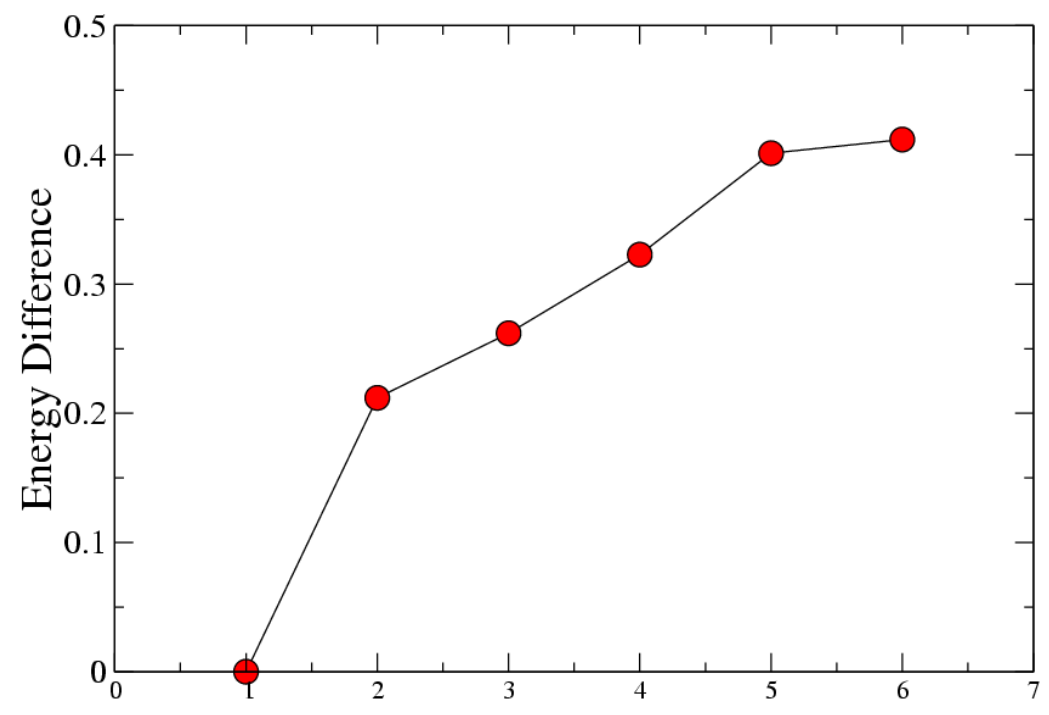

Fig.7. Energy differences for the 2x2x1 case.

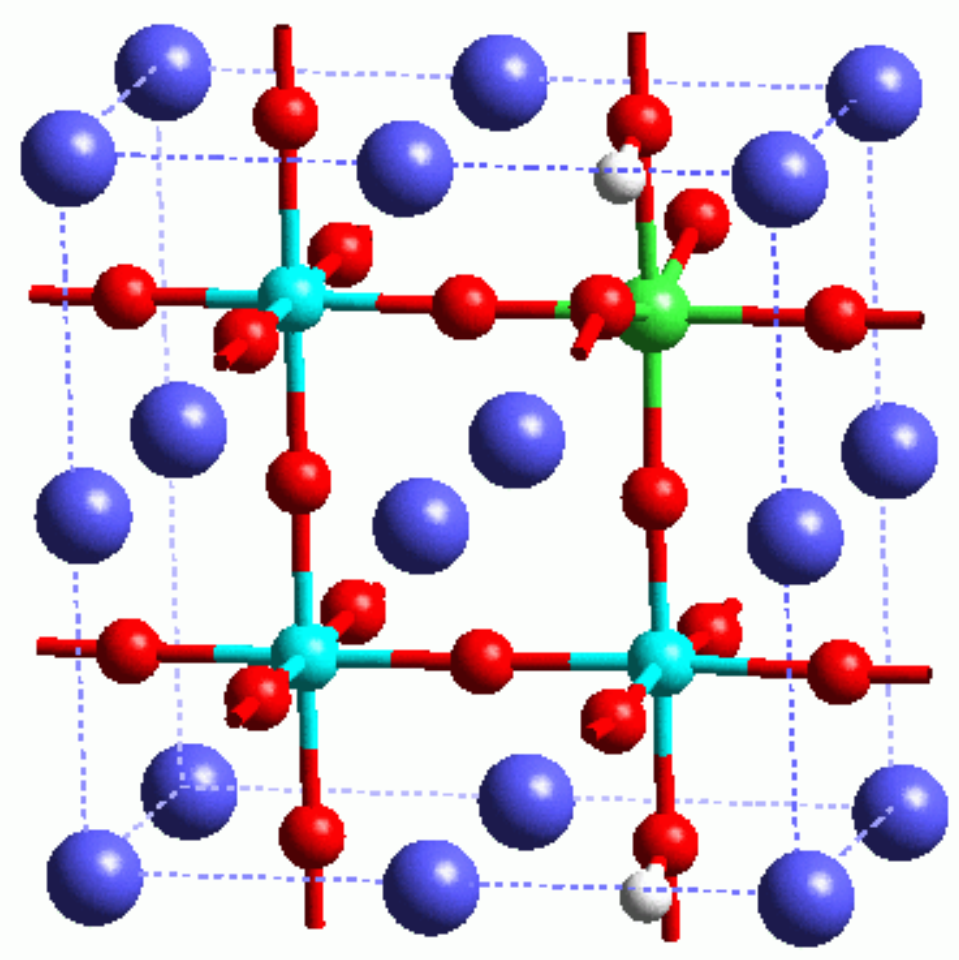

Fig.8. The lowest energy configuration for $\mathrm{BaY}_{0.25} \mathrm{Zr}_{0.75} \mathrm{H}_{0.25} \mathrm{O}_{3}$. 


\section{$\mathrm{BaY}_{0.125} \mathrm{Zr}_{0.875} \mathrm{H}_{0.125} \mathrm{O}_{3}$}

Due to the computational intensity of the calculation involving eight unit cells we have only studied two configurations for this concentration so far. Nevertheless, they are quite enlightening. In this case, the $\Delta \mathrm{E}$ between configurations, one on the $\mathrm{YO}_{6}$-octahedron and the other on the $\mathrm{ZrO}_{6^{-}}$ octahedron, is about $0.19 \mathrm{eV}$, which might indicate a rather shallow transition state energy.

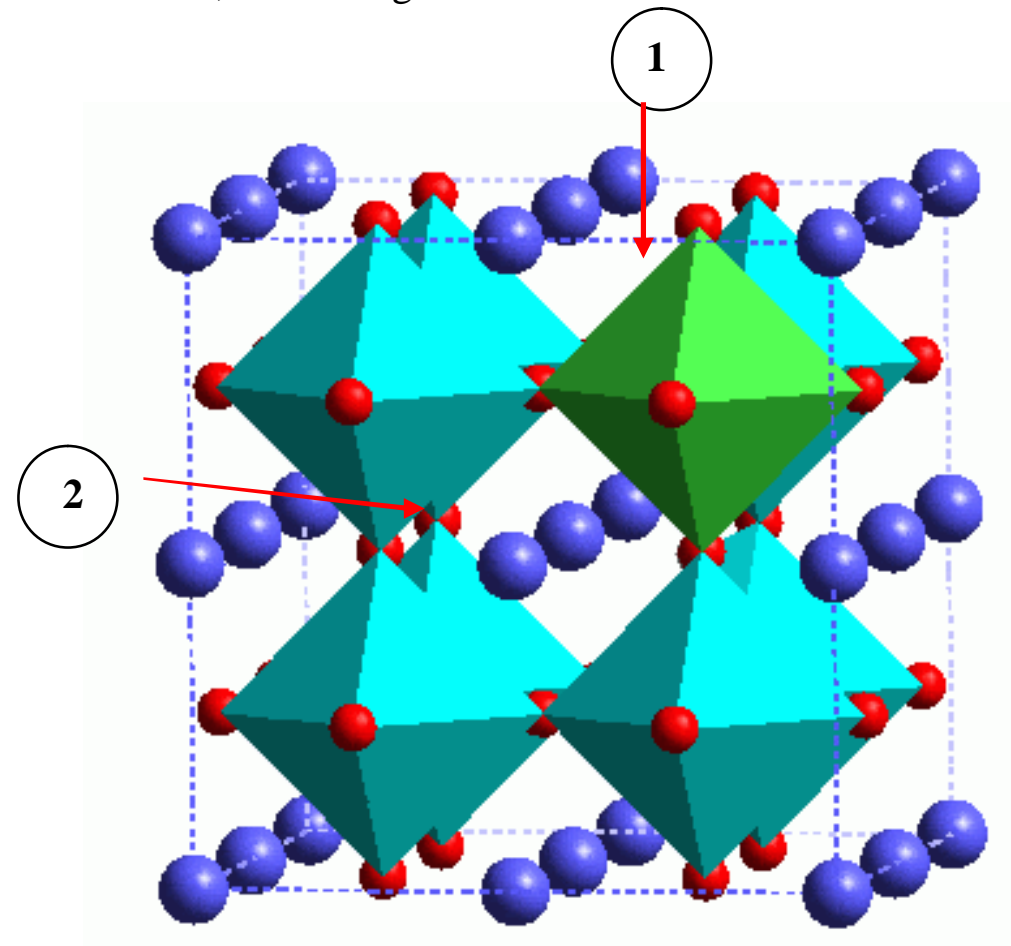

Fig.9. Schematic representation of the relaxed states considered for $\mathrm{BaY}_{0.125} \mathrm{Zr}_{0.875} \mathrm{H}_{0.125} \mathrm{O}_{3}$.

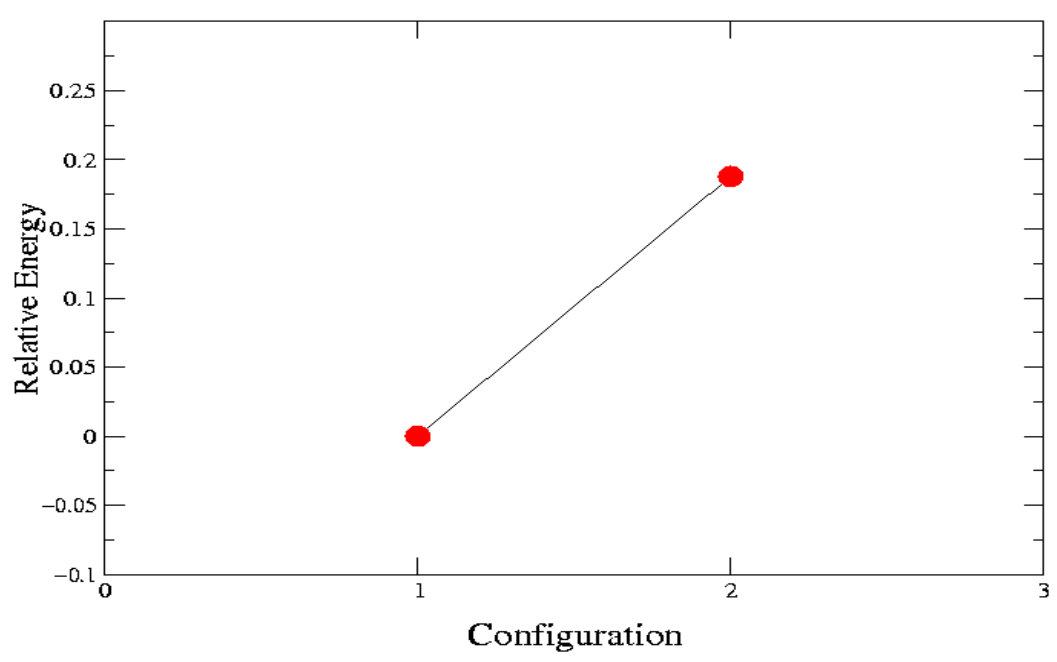

Fig.10. Energy differences for $\mathrm{BaY}_{0.125} \mathrm{Zr}_{0.875} \mathrm{H}_{0.125} \mathrm{O}_{3}$. 


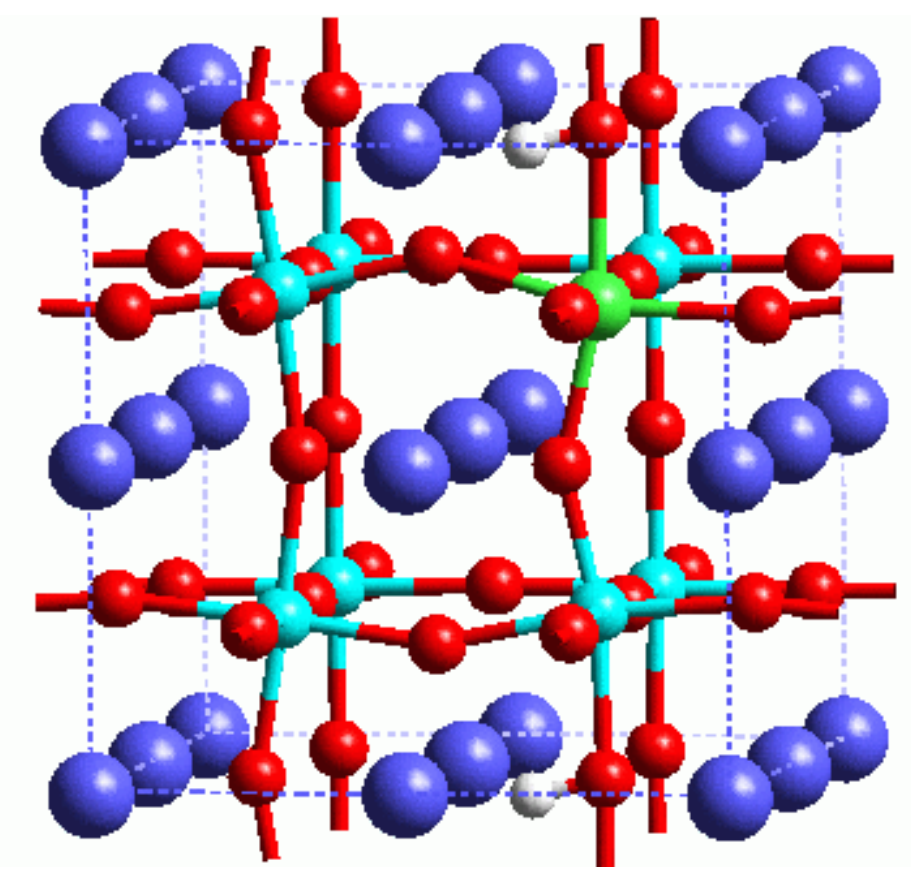

Fig.11. Relaxed configurations for $\mathrm{BaY}_{0.125} \mathrm{Zr}_{0.875} \mathrm{H}_{0.125} \mathrm{O}_{3}$.

\section{First estimate of transition barriers}

From the analysis of $\mathrm{BaY}_{0.5} \mathrm{Zr}_{0.5} \mathrm{H}_{0.5} \mathrm{O}_{3}$ it is possible to get some information about the shape of the transition barrier when a proton moves along an edge of the oxygen octahedron parallel to the plane defined by the yttrium atoms. This estimation is obtained by comparing the energies at points 1, 2 and 3 in Fig.3. Fig.12 shows this comparison.

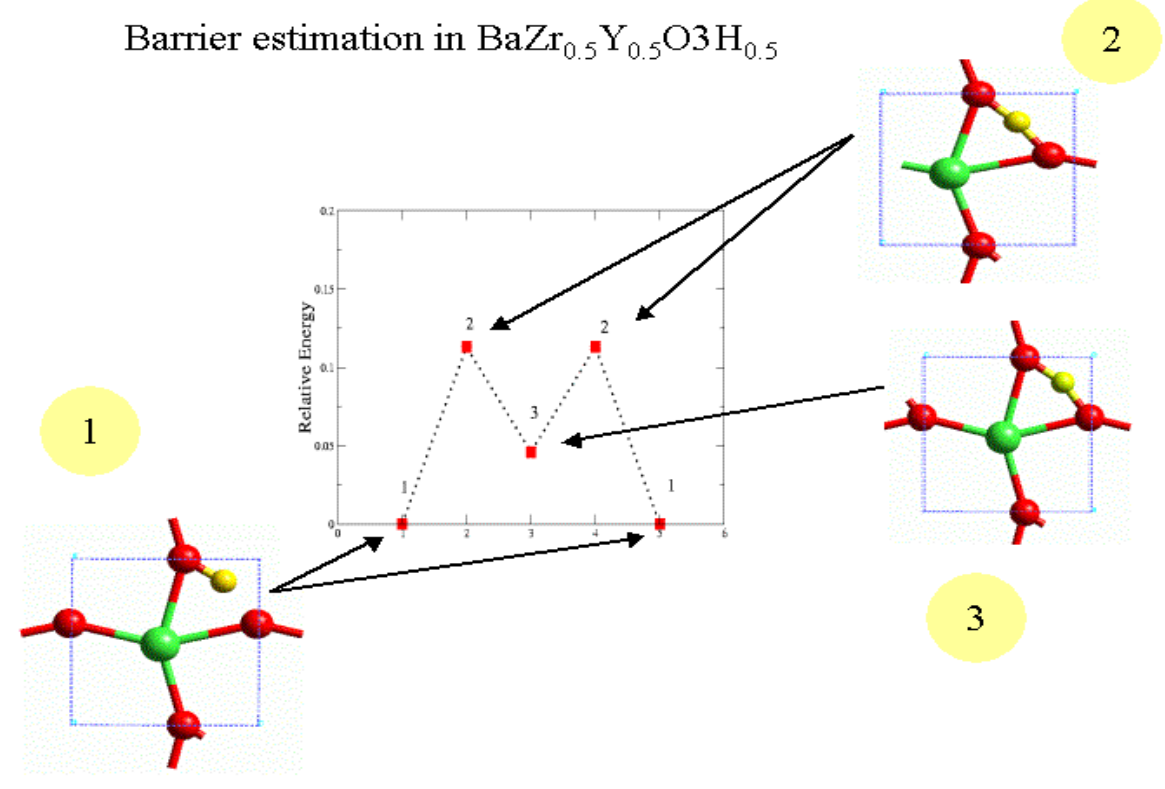


Fig.12. Relative energies of the relevant states along the path on the edge of the oxygen octahedron parallel to the Y plane.

Fig. 13 shows the main characteristics of the states whose energies are depicted in Fig.12.

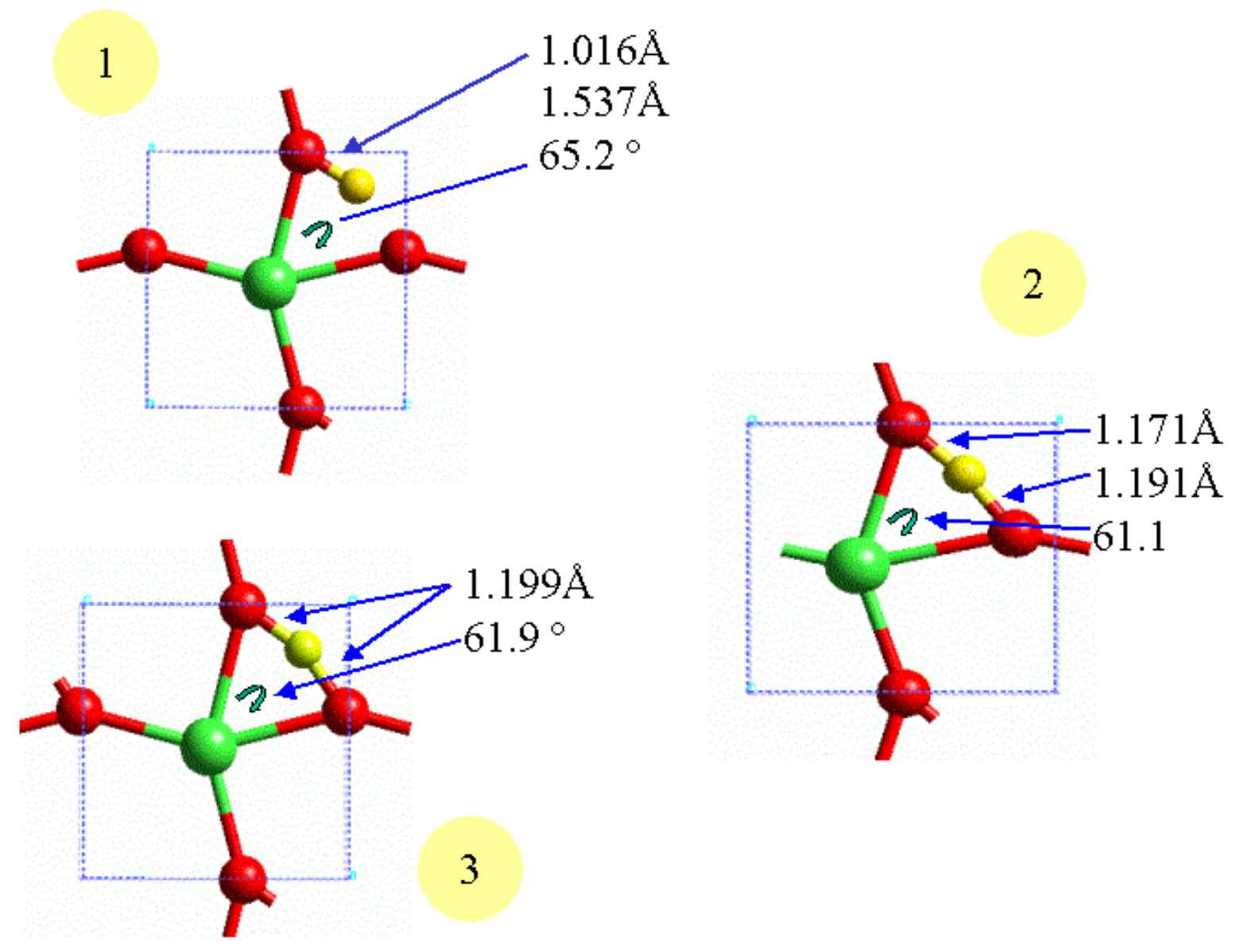

Fig.13. The main characteristics of the states.

\section{Calculations}

As mentioned above this data is being currently used to fit the parameters of the Reax Force Field. Once this task is completed MD calculations are to be performed. In order to perform NVT trajectories and appropriate algorithm is to be used. In this sense, taking into account that fluctuations might play a crucial role, we have constructed a MD code based on Nose-Hoover formalism [6]. Test runs performed on simple 1000 particles 3D L.J. system give results than can be properly described saying that the resulting velocity distribution function can be characterized by $\langle\mathrm{v}\rangle=0.00,(2 / 3)\langle\mathrm{Ke}\rangle /\left(\sigma^{2}\right)=0.0001$, Skewness $\cong 0.0001$ (which measures the symmetry of the distribution function) and the Kurtosis excess $\cong 0.0001$. 


\section{CONCLUSIONS}

$\mathrm{Ab}$ initio calculations of the $\mathrm{EOS}$ for $\mathrm{BaZrO}_{3}$ have been performed and the bulk modulus has been obtained. The value of the modulus is in good agreement with reported experimental values.

Equilibrium proton positions in Y-doped $\mathrm{BaZrO}_{3}$ with different concentrations of the dopant have been investigated. The general features of these positions confirm the result of the previous calculations. The high concentration of the dopant might lead to a trapping of the protons.

The first estimate of the transition barriers has been performed. From our results based on the ab initio calculations we found that the shape of the energy difference landscape showed two maximums (symmetric with respect to the center of the path). This is in contrast with the previous reported results, in which the transition barrier displayed just one maximum at the center.

In the forthcoming months we will perform the following calculations:

- We will continue exploring the energy landscape for the equilibrium positions of protons. We will explore possible diffusion routes from a QM analysis.

- We will incorporate the ReaxFF into a Nose-Hoover MD in order to investigate the dynamics of proton diffusion and to determine diffusion constants.

- We will explore the possibility of mapping of the proton diffusion process on an $\mathrm{n}$ order percolation problem.

- We will start analyzing the implementation of a Kinetic Monte Carlo code.

\section{REFERENCES}

1. W.Munch, K.D. Kreuer, St. Adams, G.Seifert and J.Maier, Phase Trans. 68567 (1999).

2. R.A.Davis, M.S. Islam and J.D. Gale Solid State Ionics 126323 (1999).

3. P.Hohenberg and W.Kohn, Phys. Rev. 136 864B (1964).

4. W.Kohn and L. J. Sham, Phys. Rev. 1401133 A (1965).

5. J. P. Perdew K.Burke and M. Ernzerhof, Phys. Rev. Lett. 773865 (1996).

6. W.G.Hoover Phys. Rev.A 311695 (1985). 


\section{LIST OF ACRONYMS AND ABBREVIATIONS}

SOFC - Solid Oxide Fuel Cell, QM - Quantum Mechanics, EOS - Equation of States,

ReaxFF - First Principles-Based Reactive Force Field, MD - Molecular Dynamics,

KMC - Kinetic Monte Carlo, DFT - Density Functional Theory, GGA - Generalized Gradient Approximation, NVT - Constant-volume/Constant-temperature dynamics. 